\title{
A Wearable Loop-Dipole Combined Antenna
}

\author{
Qing-Nian Zhang ${ }^{1}$, Ji Zhang ${ }^{1}$, Dan-Yang Zhang ${ }^{1}$, Wen-Jun Lu ${ }^{1 *}$, Kin Fai Tong ${ }^{2}$, Hong-Bo Zhu ${ }^{1}$ \\ 1. College of Telecommunications and Information Engineering, Nanjing University of Posts \&Telecommunications, Nanjing, \\ 210003, China \\ 2. Department of Electronic and Electrical Engineering, University College London, UK \\ *wjlu@,njupt.edu.cn
}

\begin{abstract}
The design approach of a loop-dipole combined antenna for wearable applications is proposed. In order to mitigate the antenna's performance deterioration caused by the human body, additional loops are incorporated with a wideband dipole. The loops can i) widen the impedance bandwidth by minimizing the reactance of the antenna, ii) increase the antenna's directivity and iii) act as wearable accessories for easy mounting to the users' bodies as well. Then, the interaction between the antenna and the human body can be reduced. The antenna is simulated and measured for both free space and on-body cases. Operation bandwidth of $40 \%\left(20 \log \left|\mathrm{S}_{11}\right|<-10 \mathrm{~dB}\right)$ from 6 to 9 $\mathrm{GHz}$ is obtained. The measured results show good agreement with the simulated ones.
\end{abstract}

Index Terms-Loop-dipole combined antenna, wearable antenna.

\section{INTRODUCTION}

In recent years, with the rapid development of broadband wireless communications, there has been a considerable increase of interest in wireless body area networks (WBAN) applications [1]. Most researches have been directed toward UWB technology, and numerous wearable wideband antennas have been developed [2-4]. Besides the wideband antennas, most presented body-worn antennas are omnidirectional [4-5] or narrowband [6-7]. However, the human body tends to significantly affect the antennas' performances, hence antenna designs with directional radiation beams and less sensitive to the human body are highly desirable [8-10]. Basically, unidirectional antennas minimize the effects caused by the close proximity of the human body and body exposure to EM radiation [6] in WBAN. To reduce the interaction between the antenna and human body, usually a metallic reflector is needed to isolate the radiator and the human body from each other. Thus, patch antennas [6], textile antennas [11] or multi-layer antennas [7] with a metallic reflector are suitable for wearable applications. Due to their inherent structures, most of these wearable antenna's bandwidths are usually narrow and not suitable for high data-rate applications. As an alternative way, wearable antennas placed on AMC [12] or EBG surfaces [13] exhibit good immunity to the human body. However, metasurface is complex and expensive the cost of the wearable antenna would be significantly increased, but the operation bandwidth might possibly be decreased due to the frequency selectivity characteristic of meta-surfaces. Therefore, it is challenging to design a wideband wearable antenna with human body independency, simple structure and low cost.

In this paper, design approach of a new wearable, lowprofile loop-dipole combined antenna is proposed and verified based on the asymmetric dipole antenna reported in [14]. When a dipole and a loop antenna are properly combined and excited, because of their complementary reactance relation, the resultant reactance of the combined antenna can be very close to zero. Therefore a wideband operation can be achieved [15]. At the same time, uni-directional radiation pattern can also be obtained owning to the Huygens source effect [15]. In the way, the interaction between the antenna and the human body can be mitigated. Moreover, the loop elements of the antennas can be utilized as accessories for mounting the wearable devices to the users' bodies as well.

\section{OPERATION PRINCIPLE AND PRELIMINARY RESULTS}

Conceptual design of the complementary wearable antenna initiated from a symmetrical circular dipole is shown Fig.1. Since the near field and reactance characteristics of the complementary antenna have been intensively discussed in [15], such as the equivalent circuit model and the resonant condition of the complementary dipoles, the far field characteristic is analyzed in this paper. As shown in Fig. 1, a dipole can be considered as a virtual time-harmonic, $y$-oriented electric dipole $\mathbf{J}$ in free space that locates at the origin of coordinate system. The additional loop is equivalent to a fictitious, $x$-oriented magnetic dipole M. When the dipole and the loops are co-located and excited by signal with equal amplitude and in-phase, an unidirectional loop-monopole combined antenna can be achieved.

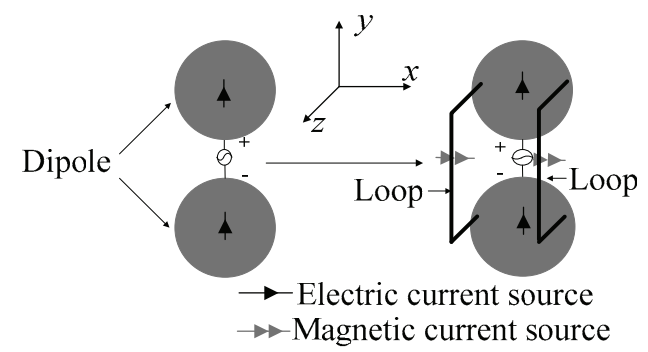

Fig.1. Equivalent sources model.

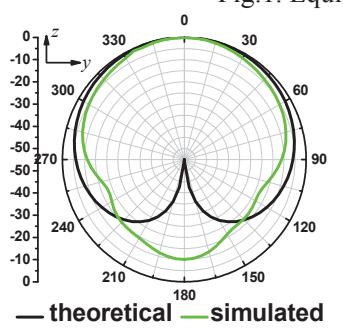

(a)

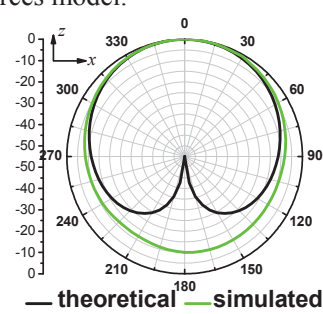

(b)
Fig.2. Theoretical and simulated radiation patterns (a) E-plane, (b) H-plane.

For brevity and comparison, an initial prototype antenna on air substrate with two loops is simulated by using Ansoft's HFSS. The initial antenna is a symmetrical circular dipole with 
radius of $10 \mathrm{~mm}$, loop length, height, thickness of $20 \mathrm{~mm}, 10$ $\mathrm{mm}, 4 \mathrm{~mm}$, respectively. The theoretical and the simulated radiation patterns are plotted and compared in Fig. 2. It can be observed that a +z-oriented, cardiac-shaped beam can be achieved. It is noted that the mutual coupling effects between the sources are not considered in the ideal theoretical model. Therefore, the two results show discrepancy in the back lobe and the radiation null (the-z-direction). Good agreement in the main lobes can be observed. Thus the operation principle of the loop-combined antenna is theoretically validated.

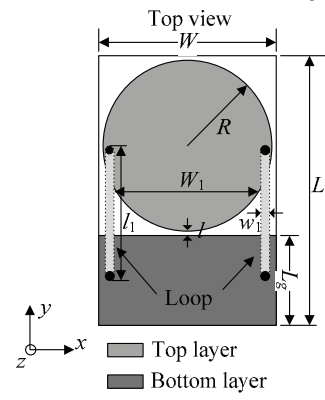

Side view

Fig.3. Geometry of the proposed antennas.

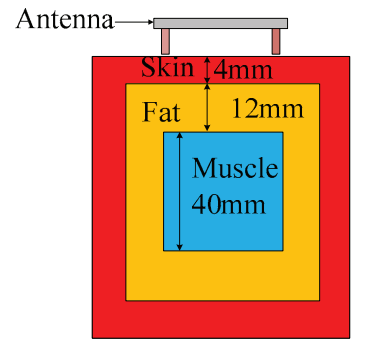

(a)
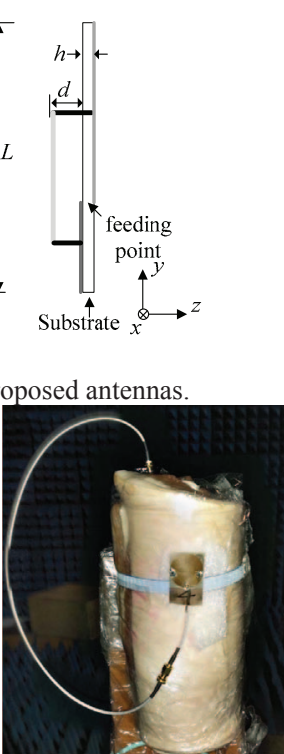

(b)

Fig. 4(a) Model of the phantom, and (b) measurement setup in anechoic chamber.

Then, a modified loop-dipole combined antenna is designed and shown in the Fig. 3. Its parameters are tabulated in Table I. For better impedance matching, one arm of the circular dipole is replaced by a rectangular patch. It is fabricated on a piece of Roger's 5880 substrate with relative permittivity $\varepsilon_{r}=2.2$, loss tangent $\tan \delta=0.0009$ and thickness $h=0.127 \mathrm{~mm}$. The antenna is fed by a $50 \Omega$ coaxial cable with its inner conductor connected to the circular patch, and with its outer shield conductor adhesively bonded to the rectangular patch. Additionally, running a flexible belt through the two loops can fix the antenna on clothes or human bodies.

TABLE I

PARAMETERS OF THE ANTENNA, UNIT: MM

\begin{tabular}{c|c|cc|}
\hline \hline Parameters & Value & Parameters & Value \\
\cline { 2 - 4 }$W$ & 28 & $L_{g}$ & 14 \\
\hline$R$ & 14 & $L$ & 45 \\
\hline$l$ & 0.3 & $l_{1}$ & 18 \\
\hline$h$ & 0.127 & $d$ & 3 \\
\hline$w_{1}$ & 1 & $W_{l}$ & 24 \\
\hline \hline & HUMAN BODY CHARACTERISTICS AT 6 GHz & \\
\hline \multirow{2}{*}{ Layer } & Relative & Conductivity & Thickness \\
& permittivity & (S/m) & (mm) \\
\hline Skin & 34.95 & 3.89 & 4 \\
Fat & 4.95 & 0.31 & 12 \\
Muscle & 48.22 & 5.20 & 20 \\
\hline
\end{tabular}

The antenna is simulated by employing Ansoft's HFSS to evaluate its performance when located on a human arm. A simplified three-layered phantom model as shown in the Fig. 4 (a) is used. The overall size of the phantom is $72 \times 150 \times 72$ $\mathrm{mm} 3$. The phantom is composed of skin, fat and muscle $[6,9]$, while the other tissues are not considered. The proposed antenna was supported by a foam (with a relative permittivity of about 1.1) layer of $1 \mathrm{~mm}$ on the phantom when the radiation patterns were measured in an anechoic chamber. The electrical parameters and thickness of different tissues at $6 \mathrm{GHz}$ [16] are tabulated in Table II.

\section{ANTENNA PROXIMITY TO HUMAN BODY}

The $\left|\mathrm{S}_{11}\right|$ of the prototype antenna is measured by using an Agilent's 8720ET vector network analyzer. Both the free space and on-phantom cases are studied. As shown in the Fig. 5 (a) and (b), it can be observed that the simulated and measured impedance bandwidth of the proposed antenna is almost stable in the range from 6 to $9 \mathrm{GHz}$ no matter if it is mounted on the phantom or in free space.

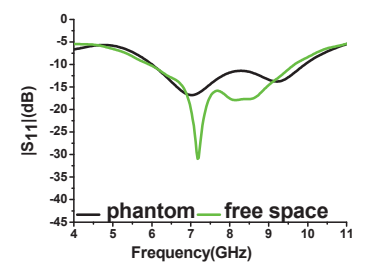

(a)

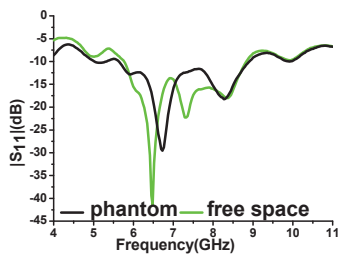

(b)
Fig.5. Simulated and measured $\left|\mathrm{S}_{11}\right|$ of the proposed antennas: (a) simulated $\left|S_{11}\right|$, (b) measured $\left|S_{11}\right|$

The radiation patterns and gain of the proposed antenna are measured by using the NSI's NSI-800F-10x antenna measurement system. As shown in Fig. 4(b), the prototype antenna is mounted on a fresh pork phantom with size of $450 \times 75 \times 75 \mathrm{~mm}^{3}$ with a flexible, plastic belt passed through the two loops. The thickness of the skin, fat and muscle of the pork phantom is $4 \mathrm{~mm}, 14 \mathrm{~mm}$ and $21 \mathrm{~mm}$, respectively. There is a $1 \mathrm{~mm}$ foam-filled gap between the skin and the antenna.

The radiation patterns in both principal planes at 6,7 , and 8 $\mathrm{GHz}$ are plotted and compared in Fig. 6. It is observed that the antenna exhibits a +z-oriented radiation pattern within its operating frequency range. The measured patterns agree well with the simulated ones, especially in the main lobe. The measured cross-polarization levels are higher than the simulated ones, while they are still about $20 \mathrm{~dB}$ lower than the co-polarization, as shown in Fig. 7. All of the measured results of phantom agree well with the measured ones in free space.

The simulated and measured peak gains and simulated efficiency of the antenna on the phantom are plotted in Fig. 7. The in-band average measured gain is $1.82 \mathrm{~dB}$, which agrees reasonably with the simulated result of $2.00 \mathrm{~dB}$ and the average simulated efficiency is $37.6 \%$. The gain degradation at 6.5 $8.5 \mathrm{GHz}$ frequency band is caused by the frequency dispersive characteristic and the electromagnetic loss of the real phantom tissues. While in the simulation, such frequency dispersions are 
not considered. Due to the discrepancies of the electrical parameters, i.e., relative permittivity and conductivity, between the empirical values [16] and that of the phantom, and the errors in the measurement, the measured gain-frequency curve shows quite similar trend with the simulated one with some small variations. However, a moderate measured gain of $1.8 \mathrm{~dB}$ with flatness less than $2 \mathrm{~dB}$ is still obtained within 6 - $9 \mathrm{GHz}$.

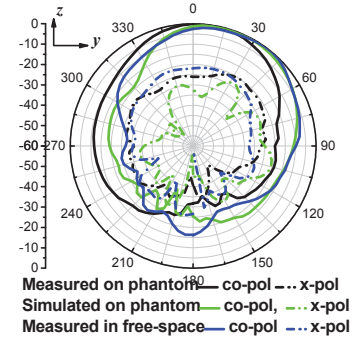

(a)

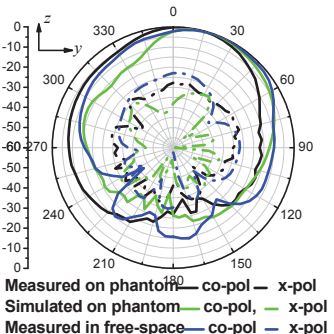

(c)

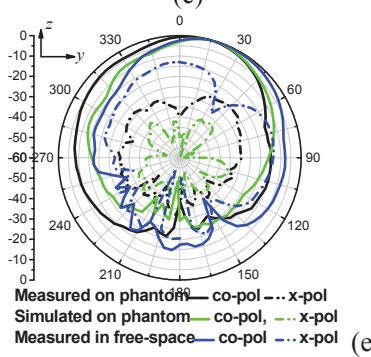

Measured in free-space - co-pol..$- x$-pol

Fig.6. Radiation patterns of the proposed antenna: (a) $y z$-plane, $6 \mathrm{GHz}$, (b) $z x$-plane, 6GHz, (c) $y z$-plane, 7GHz, (d) $z x$-plane, 7GHz, (e) $y z$-plane, $8 \mathrm{GHz}$, (f) $z x$-plane, $8 \mathrm{GHz}$.

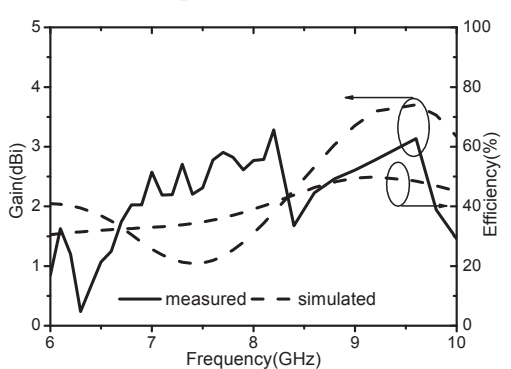

Fig.7. Measured and simulated peak, realized gain.

\section{CONCLUSION}

A wearable complementary, loop-dipole combined antenna is proposed and studied in this work. Loop structures are introduced to minimize the near field stored energy, generate a unidirectional cardiac-shaped radiation pattern and then lead to a new wearable complementary antenna with lower human body dependency. In addition, the loops act as wearable structures as well. The operation principle of the antenna is first explained. Then, prototypes are designed, fabricated and measured to verify the operation principles, design approach and numerical results. The proposed wearable antenna has good impedance matching and stable unidirectional radiation characteristics from 6 to $9 \mathrm{GHz}$. It has a simple structure and is easy to fabricate. Therefore, it is suitable for potential wideband, wearable applications for the next generation wireless communications.

\section{ACKNOWLEDGEMENT}

This work was supported in part by the National Natural Science Foundation of China under grant no. 61471204 and 61427801.

\section{REFERENCES}

[1] P. S. Hall, Y. Hao, Y. I. Nechayev, A. Alomainy, C. C. Constantinou, C. Parini, et al. "Antennas and propagation for on-body communication systems," IEEE Antennas Propag. Mag., vol. 49, no. 3, pp. 41-58, Jun. 2007.

[2] M. Klemm and G. Troester, "Textile UWB antennas for wireless body area networks," IEEE Trans. Antennas Propag., vol. 54, no. 11, pp. 31923197, Nov. 2006.

[3] N. Chahat, M. Zhadobov, R. Sauleau, and K. Ito, "A compact UWB antenna for on-body applications," IEEE Trans. Antennas Propag., vol.59, no. 1, pp. 1123-1131, Jan. 2011.

[4] C. P. Deng, X. Y. Liu, Z. K. Zhang, and M. M. Tentzeris, "A miniascapelike triple-band monopole antenna for WBAN applications," IEEE Antennas Wireless Propag. Lett., vol. 11, pp. 1330-1333,Nov. 2012.

[5] G. Almpanis, C. Fumeaux, J. Frohlich, and R. Vahldieck, "A truncated conical dielectric resonator antenna for body-area network applications," IEEE Antennas Wireless Propag. Lett., vol. 8, pp. 279-282,May 2009.

[6] I. Locher, M. Klemm, T. Kirstein, and G. Trster, "Design and characterization of purely textile patch antennas," IEEE Trans. Adv. Packag., vol. 29, no. 4, pp. 777-788, Nov. 2006.

[7] A. C. Gareth, and G. S. William, "Antennas for over-body-surface communication at $2.45 \mathrm{GHz}$," IEEE Trans. Antennas Propag., vol. 57, no. 4, pp.844-855, Apr. 2009

[8] M. Klemm, I. Z. Kovcs, G. F. Pedersen, and G. Troster, "Novel smallsize directional antenna for UWB WBAN/WPAN applications," IEEE Trans. Antennas Propag., vol.53, pp. 3884-3896, Dec. 2005.

[9] A. Zaric, J. R. Costa, C. A. Fernandes, "Design and ranging performance of a low-profile UWB antenna for WBAN localization applications," IEEE Trans. Antennas Propag., vol. 62, no. 12, pp. 6420-6427, Dec. 2014.

[10] S. Lemey, F. Declercq, H. Rogier, "Dual-Band substrate integrated waveguide textile antenna with integrated solar harvester," IEEE Antennas Wireless Propag. Lett., vol.13, 269-272, 2014.

[11] B. P. Samal, J. P. Soh, G. A. E. Vandenbosch, "UWB all-textile antenna with full ground plane for off-body WBAN communications," IEEE Trans. Antennas Propag., vol.62, no.1, pp. 102-108, Jan. 2014.

[12] H. R. Raad, A. I. Abbosh, H. M. Al-Rizzo, and D. G. Rucker, "Flexibleand compact AMC based antenna for telemedicine applications," IEEE Trans. Antennas Propag., vol. 61, no. 2, pp. 524531, Feb. 2013.

[13] S. Z. Zhu and R. Langley, "Dual-band wearable textile antenna on an EBG substrate," IEEE Trans. Antennas Propag., vol. 57, no. 4, pp.926935, Apr. 2009.

[14] S. Barbarino and F. Consoli, "Study on super-wideband planar asymmetrical dipole antennas of circular shape," IEEE Trans. Antennas Propag., vol. 58, no. 12, pp. 4074-4078, Dec. 2010.

[15] K. M. Luk, and B. Q. Wu, "The magnetoelectric dipole-a wideband antenna for base stations in mobile communications," Proc. IEEE, vol. 100, no. 7, pp. 2297-2307, Jul. 2012.

[16] 2013, Dielectric Properties of Body Tissues [Online]. Available: http://niremf.ifac.cnr.it/tissprop/ 